\title{
The Discrete Paired-trial Variable-delay T-maze Task to Assess Working Memory in Mice Gian Marco Leggio ${ }^{1, *}$, Sebastiano Alfio Torrisi ${ }^{1}$ and Francesco Papaleo ${ }^{2, *}$
}

\author{
1Department of Biomedical and Biotechnological Sciences, University of Catania, Catania, Italy; \\ ${ }^{2}$ Genetics of Cognition laboratory, Neuroscience area, Istituto Italiano di Tecnologia, Genova, Italy \\ *For correspondence: gianmarco.leggio@unict.it; francesco.papaleo@iit.it
}

\begin{abstract}
[Abstract] Working memory abnormalities involving the prefrontal cortex (PFC) dramatically contribute to poor functional outcomes in patients with schizophrenia and still represent an unmet therapeutic need. Studies in rodents might provide essential tools to understand the mechanisms underlying PFC-dependent working memory dysfunctions, as well as precious tools for genetic and pharmacological testing. However, proper tests assessing working memory and sensitive to PFC-dependent functions must be used. In this regard, the discrete paired-trial variable-delay T-maze task, equivalent to delayed non-match to sample tasks used in humans, has proved to be an effective paradigm to test PFC-dependent working memory dysfunctions with high predictive validity in human studies.
\end{abstract}

Keywords: Discrete paired-trial variable-delay T-maze, Working memory, Animal models, Cognitive dysfunctions, Schizophrenia

[Background] The term working memory refers to the type of memory that is active and relevant only for a short period of time, on the scale of seconds, while performing complex tasks such as reasoning, comprehension and learning. The concept of working memory evolved from that of short-term memory and now it stands at the interface between perceptual processes and long-term memory formation. The major components of working memory, as suggested by Baddeley's model (Baddeley, 2010), are: i) a short-term storage buffer for visual-spatial information that provides a virtual environment for physical simulation, calculation, visualization and optical memory recall (often referred as the visuo-spatial scratch pad); ii) a short-term storage buffer for verbal information (referred to as the phonological loop); (iii) a central executive component that is responsible for response selection and for coordinating the outputs of different short-term memory buffers; iv) an episodic buffer, in which complex multimodal events are integrated and stored online. In this model, the maintenance of specific information is governed by the buffer systems, while the regulation and coordination of this information (i.e., updating and maintenance of task goals, management of interference, and manipulation and transformations of stored content) are handled by the central executive processes. Impairments across the domains of phonological, visuo-spatial and central executive working memory are among the most consistently cognitive deficits observed in patients with schizophrenia (Castner et al., 2004; Forbes et al., 2009). The working memory central executive component has been associated in many studies with the function of the dorsolateral PFC. Instead, the storage buffers are thought to depend more on the inferior frontal cortex and parietal cortical areas (Wager and Smith, 2003). The remarkable correspondence between 
performances of human patients with frontal lobe lesions, PFC-lesioned monkeys and rodents and schizophrenic patients made the PFC-dependent tasks among the most used in behavioral/fMRI studies in schizophrenia and relative translational preclinical research (Callicott et al., 2000; Kellendonk et al., 2006; Papaleo et al., 2008; Barch et al., 2012).

There are numerous working memory tasks that have been employed and validated in rodents in order to reliably measure the maintenance of visuo-spatial information (Dudchenko, 2004; Kellendonk, et al., 2006; Papaleo et al., 2014), for example, the 8-arm radial maze "delayed non-match to sample" or "win-shift" (Seamans et al., 1995; Seamans and Phillips, 1994), the 8-arm maze "random foraging task" (Floresco et al., 1997; Seamans et al., 1995), the odor span tasks (Dudchenko, 2004; Young et al., 2007) and some paradigms of delayed matching and delay non-matching to sample position operant conditioning tasks (Dunnett, 1993). These tasks involve an initial "sample" or "forced run" phase in which the rodent is exposed to a visual target or an arm of the maze. Subsequently, in the "choice" phase that is run after a variable delay, the subject is simultaneously presented with the original sample (the "match") and another visual target or arm (the "non-match"). These pairs of phases must be presented repeatedly but importantly, with randomly changing cues presented in the sample phase. Thus, the working memory construct is based on the fact that the tested rodent is required to integrate information held online (the sample phase) with the learned rule (non-match or match to sample). This paradigm has been mostly implemented in mice using T-mazes (Aultman and Moghaddam, 2001; Kellendonk et al., 2006). In this context, the discrete paired-trial variable-delay T-maze task seems to be similar to the human delayed response task and also it relies on mPFC functions (Kellendonk et al., 2006). Indeed, it is based on the delayed non-match to position paradigm where the delayed alternations responses are driven by food reinforcement (Ji et al., 2009; Leggio et al., 2019). In particular, during a "sample" or "forced run", the experimental subject is forced to explore an arm of the maze. Then, after a variable delay, in the "choice run" phase the subject has to choose between the original sample (the "match") and the opposite arm (the "non-match"). Rodents are then presented with a sequence of randomly chosen forced runs, each followed by a choice run. The working memory construct is based on the fact that the experimental animal is required to integrate information held online (the forced run) with the learned rule (non-match or match to sample). In this context, the role of PFC for supporting complex executive functions is well acknowledged (Scheggia et al., 2018 and 2020), as well as the necessity of the hippocampus involvement for different tasks used to study the spatial working memory, such as the Radial Arm Maze (Myroshnychenko et al., 2017) and the Morris Water Maze (Morris et al., 1982; Dudchenko, 2004).

\section{Materials and Reagents}

1. Male C57BL6/J mice (8-16 weeks old, Charles River Laboratories Italia, Italy)/Genetic mouse models

Note: It is also possible to use female C57BL6/J mice (8-16 weeks old) after evaluation of estrous state as well as other mouse strains without any locomotor impairment. 
2. Food reinforcer (14 mg, 5TUL, TestDiet, Richmond, IN)

3. Ethanol $10 \%$

\section{Equipment}

1. The T-maze apparatus

The T-maze apparatus was built out of transparent Plexiglass $(0.5 \mathrm{~cm}$ thick; dimensions of the alleys: $40 \times 10.2 \times 17.5 \mathrm{~cm}$. Light levels will be: $20 \pm 2$ lux in the main alley; and $10 \pm 2$ lux in the side alleys). A recessed cup at the end of each side alley concealed the food reinforcement from view. In addition, care was taken to remove all visual cues that could be used by the animal to guide his choice: behavioral studies were carried out in a room without any visual landmarks or windows (Figure 1).

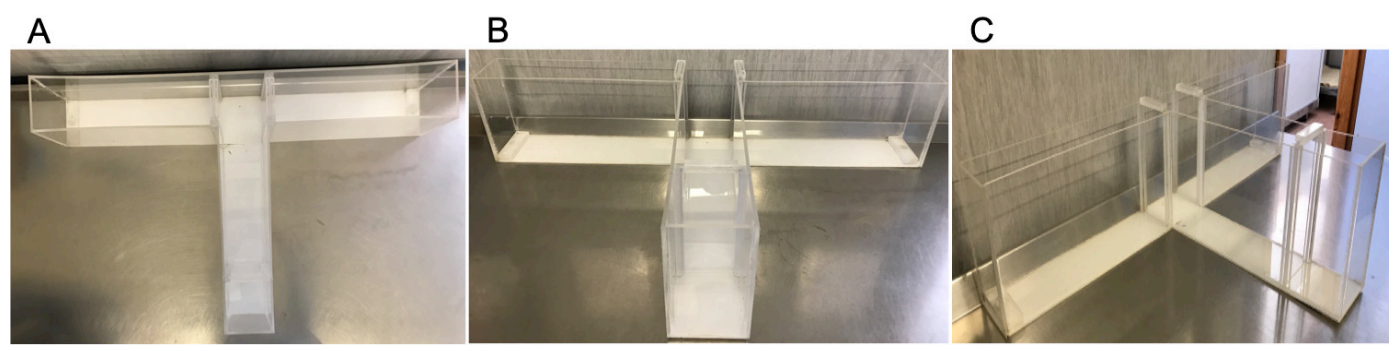

Figure 1. T-maze apparatus. Photographs show the top view (A), front view (B) and side view (C) of the T-maze for mice used in Leggio et al. (2019).

\section{Software}

1. GraphPad Prism software (GraphPad Software, USA) was used to perform the statistical analysis and artwork.

\section{Procedure}

In the discrete paired-trial variable delay T-maze task, mice were exposed to a sequence of randomly chosen forced runs, each followed by a choice run so that they were required to integrate information held online (the forced run) with the learned rule (non-match to sample) (Papaleo et al., 2012). After a week of single housing, body weight and 24-h food intake are recorded for 3 consecutive days. Animals are then food restricted throughout the experiment to maintain $90 \%$ of their ad libitum body weight. During the first week of food-restriction, each animal is also habituated to the food reinforcer (14 mg, 5TUL, TestDiet, Richmond, IN) for three consecutive days. Thereafter, animals are habituated to the T-maze apparatus allowing to retrieve the food reinforcement for $10 \mathrm{~min} /$ day for two consecutive days. After this, animals are exposed to 1 day of 10 forced-alternation runs. The animals are placed in the T-maze with one goal arm closed off and will 
Please cite this article as: Leggio et. al., (2020). The Discrete Paired-trial Variable-delay T-maze Task to Assess Working Memory in Mice,Bio-protocol 10

have up to 2 min to locate and eat the food reinforcer in the open arm. After consuming the food pellet, each mouse is given an inter-trial period of at least $20 \mathrm{~min}$ in the home cage, and then placed back in the maze for another forced run. Beginning on the following day, the discrete-trial delayed alternation training starts. Following a randomly chosen forced run, and a 4-s delay interval in the home cage, the mouse is placed back in the maze with access to both arms. The food reinforcer is located in the opposite arm entered in the previous forced trial. After an inter-trial period of $20 \mathrm{~min}$, the animal is placed back in the maze for another forced run-choice run paired trial, for a total of ten paired trials per day. A different pseudo-randomly chosen pattern of forced runs (e.g., R-R-L-R-L-L-RL-R-L) is used every day, but on a given day the same pattern is used for all animals. The apparatus must be cleaned with water and ethanol $10 \%$ after each trial with special attention to the choice point of the T-maze. Mice are trained at a 4-s inter-run delay and 20-min inter-trial delay for 20 days, or until the mouse successfully performs 8 correct out of 10 daily trials ( $80 \%$ choice accuracy) for 3 consecutive days. Animals that do not reach this criterion are eliminated. Once the mouse performs consistently at the 4-s inter-run delay, training at three additional inter-run delays (30, 60 and $240 \mathrm{~s}$ presented in a random fashion) and a 20-s inter-trial delay begins. Mice are given 4 trials of each delay on 4 consecutive days of testing for a total of 16 trials for each day.

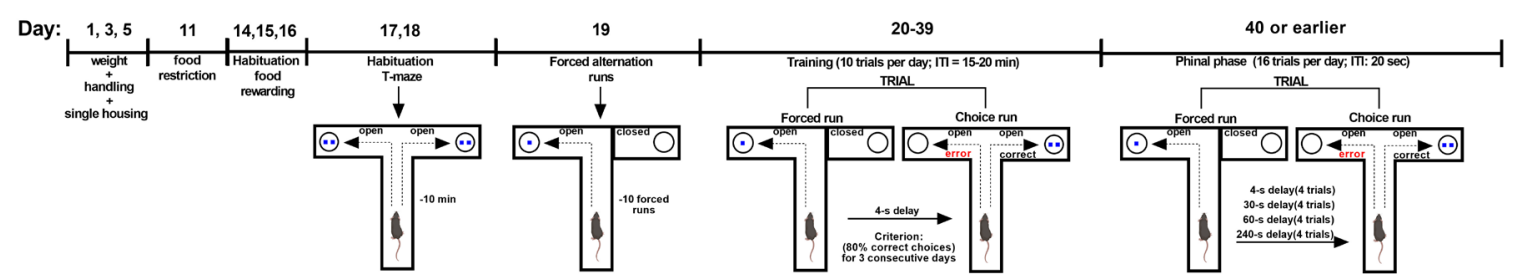

Figure 2. Timeline of the discrete paired-trial variable-delay T-maze task. Training: each discrete trial consists of a forced run-choice run pair (inter-run delay of $4 \mathrm{~s}$ and inter-trial delay of 15-20 m); Criterion: $80 \%$ of correct choices in three consecutive days (10 paired-trial/day); Final phase: intra-trial delay of $4 \mathrm{~s}, 30 \mathrm{~s}, 60 \mathrm{~s}$ and $240 \mathrm{~s}$ and inter-trial delay of $20 \mathrm{~s}$ (16 paired-trial/day for four consecutive days) (Papaleo et al., 2012; Leggio et al., 2019).

\section{Step by Step protocol}

1. Day 1: 5:00 PM: Weight and singly house each mouse. 1 min handling (H1). Change cage, water, filter and food.

2. Day 2: Singly housing habituation.

3. Day 3: Singly housing habituation. 1 min handling $(\mathrm{H} 2)$.

4. Day 4: Singly housing habituation.

5. Day 5: Singly housing habituation. 1 min handling $(\mathrm{H} 3)$.

6. Day 6: Singly housing habituation.

7. Day 7: Singly housing habituation.

8. Day 8: Singly housing habituation. 
9. Day 9: About 5:00 PM weigh the food and each mouse.

10. Day 10: About 5:00 PM, weigh each mouse and food (24 h intake)

11. Day 11: About 5:00 PM, weigh each mouse and food (24 $\mathrm{h}$ intake). Take all food off, leave water on. Change cage, filter and water.

12. Day 12: 5:00 PM, Animals are partially food-deprived and remain that way throughout the experiment. Give $\approx 60 \%$ of their $24 \mathrm{~h}$ intake. Check nest status.

13. Day 13: 5:00 PM. Give $\approx 60 \%$ of their $24 \mathrm{~h}$ intake. Arrive to $90 \%$ of their initial weight.

14. Day 14: 5:00 PM. Give $\approx 60 \%$ of their $24 \mathrm{~h}$ intake. Arrive to $90 \%$ of their initial weight. Put also the rewarding food (about 10 pellets of 5TUL $14 \mathrm{mg}$ ).

15. Day 15: 5:00 PM. Give 60\% of their $24 \mathrm{~h}$ intake. Put also the rewarding food (about 10 pellets of $14 \mathrm{mg}$ ). Arrive to $90 \%$ of their initial weight.

16. Day 16: 5:00 PM. Give $60 \%$ of their $24 \mathrm{~h}$ intake. Put also the rewarding food (10 pellets of $14 \mathrm{mg}$ ). Arrive to $90 \%$ of their initial weight.

\section{Habituation period:}

17. Day 17: Each mouse is allowed to explore the maze with all doors raised for $10 \mathrm{~min}$. Food is placed in both goal arms. Quantifying the time required to eat the $1^{\text {st }}$ pellets. They have to eat all the food presented (max 16 pellets; 2 pellets in each goal arm).

18. Day 18: Each mouse is allowed to explore the maze with all doors raised for $10 \mathrm{~min}$. Food is placed in both goal arms. Quantifying the time required to eat the $1^{\text {st }}$ pellets. They have to eat all the food presented ( $\max 20$ pellets; 2 pellets in each goal arm).

19. Day 19: Animals are exposed to 1 day of 10 forced-alternation runs. Specifically, they are placed in the T-maze with one goal arm closed off and had up to 2 min to run and eat the reward in the open arm (only one pellet in the open arm). After consuming the reward, they are removed from the maze, one trial for each mouse. Run the experiment with about 10 cages in the room. Mice are tested back-to-back. One pellet at the end of the arms.

20. Day 20-Day 39: Discrete paired-trial delayed alternation training

a. Each discrete trial consists of a forced run-choice run pair.

b. For the forced run, animals are constrained to enter a randomly chosen arm. After they consume the reward in that arm (timer started as soon as the animal has finished food) and a 4-s retention interval (this 4-s time is considered as a mean of time recorded to accomplished this procedure, see also Kellendonk et al., 2006), each mouse is placed back in the maze with access to both arms but with only the opposite arm entered in the previous forced run baited. On the forced run bait the alley with 1 pellet; on the free choice run bait the correct alley with 2 pellets. After an inter-trial period of about 15-20 min to avoid proactive interference from the last trial (Kellendonk et al., 2006), the mouse is placed back in the maze for another forced run. Test mice in the room back-to-back.

c. A different, randomly chosen, pattern of forced runs (for example, R-R-L-R-L-L-R-L-R-L) is used every day. However, on a given day the same pattern is used for all animals. Animals 
are trained at a 4-s inter-run delay for 20 days, or until they successfully perform eight out of 10 trials ( $80 \%$ correct choices) for 3 consecutive days. Animals that don't reach this criterion are rejected.

d. Light cycle: 6-6. Habituation about 8:45 AM. Test from 9:45 AM to 5:00 PM Reefed animals about 5:00 PM.

21. Day 40 or earlier: Once an animal performs consistently at the 4-s inter-run delay, training at three additional delays (30,60 and $240 \mathrm{~s}$ ) and with an inter-trial delay of only $20 \mathrm{~s}$ began. Mice are tested at all 4 inter-run delays for 4 consecutive days with 16 paired-trial/day (4 paired trial at each delay every day).

Note: This procedure is considered stressful for animals because each mouse is consecutively tested in several trials (16-paired trials) for, at least, $45 \mathrm{~min}$. Conversely, during the training phase of the paradigm, each mouse performs 10-paired trials per day with an inter-trial-interval of $10 \mathrm{~min}$.

\section{Data analysis}

1. All data generally assume a normal distribution and then they are subjected to parametric tests (one- or two-way analysis of variance (ANOVA) and two-way ANOVA with repeated measures when appropriate).

2. The Grubbs test can be performed to identify outliers (Leggio et al., 2019, Supplementary Information).

3. Latency to eat the $1^{\text {st }}$ pellets (in seconds, Days $17-18$ ) is analyzed typically via two-way repeated measures (RM) ANOVA to reveal main effects and interaction (e.g., genotype $\times$ day) followed by a suitable post-hoc test for pairwise comparisons such as the Newman-Keuls test. Days to reach criterion (in days, Days 20-39) during the training phase can be analyzed through an unpaired (two sample) t-test or a one-way ANOVA to reveal main effect (e.g., genotype or treatment) according to the number of experimental groups (two of more). Finally, percentage of correct choices (as index of working memory) during the final phase (day 40 or earlier) is also generally analyzed through two-way RM ANOVA to reveal main effects and interaction (e.g., genotype $\times$ delay), followed by an appropriate post-hoc test.

4. Graphs in Figure 3 display Latency to eat (A), Days to criterion (B), and correct choices $(\%, C)$ that wild-type (WT) and knockout mice littermates (here Dys ${ }^{+/}$mice) performed during the task (adapted from Leggio et al., 2019). Both groups learned similarly to run quickly into the maze to retrieve the reward, as indicated by the significant decrease of the latency to eat during the second day (Day 18) of exposure to the reward compared to the first day (Day 17). Moreover, both groups required the same number of days to reach the criterion of $80 \%$ of correct choices for 3 consecutive days. However, although both groups exhibited delay-dependent behavior (progressive increase of errors with longer delays), Dys ${ }^{+-}$mice showed significant working memory deficits than WT mice at both 4 and $30 \mathrm{~s}$ intra-run delays. 

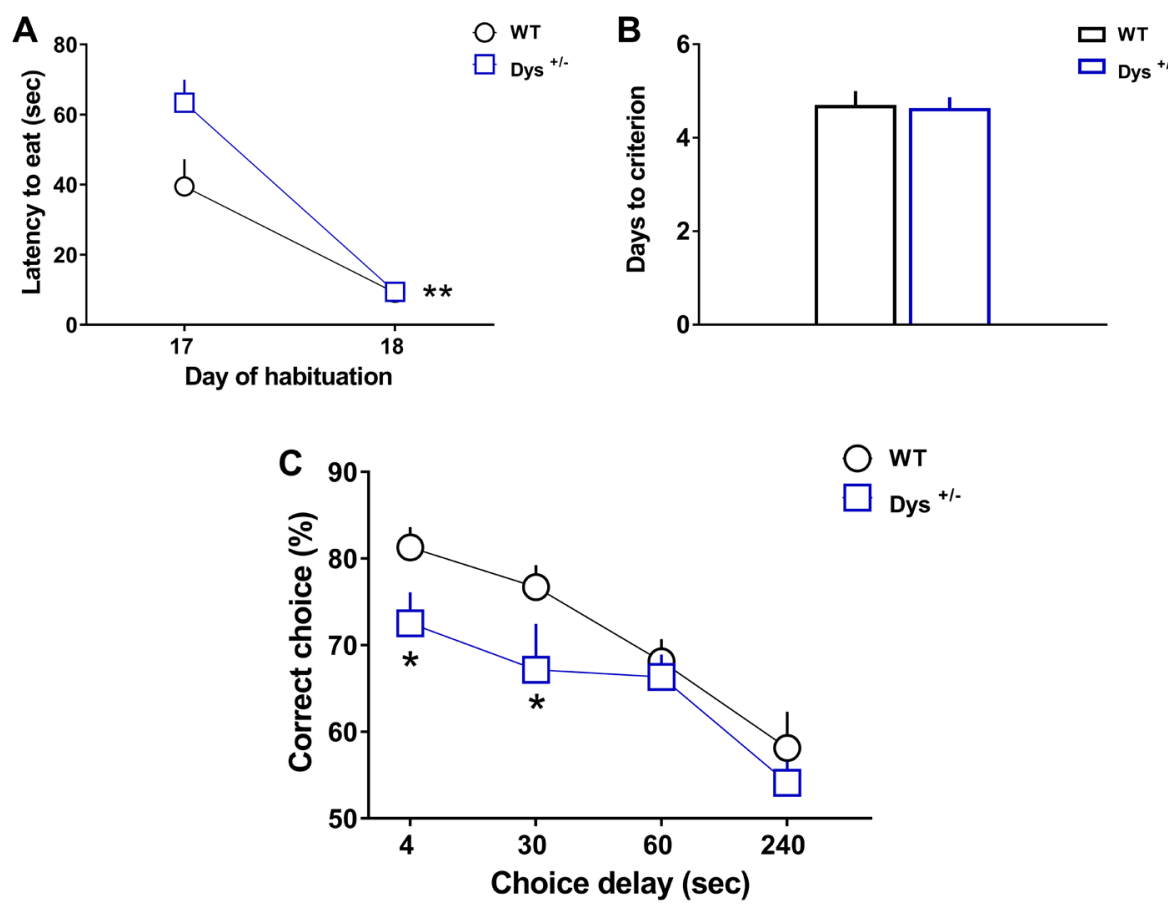

Figure 3. WT $(n=12)$ and Dys $^{+/-}(n=10)$ mice were tested in the discrete paired-trial variable-delay T-maze task. A. Latency to retrieve the reward (Genotype $\mathrm{F}_{(1,20)}=4.982, P=$ 0.0372; Day $\mathrm{F}_{(1,20)}=68.04, P<0.0001$, Genotype $\times$ Day $\mathrm{F}_{(1,20)}=5.37, P=0.031 ;$ Post hoc: ${ }^{* *} P$ $<0.01$ vs. Day 17). B. Days needed to reach the criterion $(P=0.88)$. C. Percentage of correct choices with different randomly presented intra-run delays $(4,30,60$, and $240 \mathrm{~s})$ and an inter-trial delay of $20 \mathrm{~s}$ (Genotype $\mathrm{F}_{(1,20)}=5.95, P=0.0241$; Delay $\mathrm{F}_{(3,60)}=14.93, P<0.0001$, Genotype $\times$ Delay $\mathrm{F}_{(3,60)}=0.65, P=0.58$; Post hoc: ${ }^{*} P<0.05$ vs. each WT time-point). The values are the means \pm S.E.M. (adapted from Leggio et al., 2019).

5. Graphs in Figure 4 display the data distribution of C57BI6/J WT mice $(n=46)$ obtained by clustering different experiments. Latency to eat (A), Days to criterion (B), and correct choices $(\%, \mathrm{C})$ that C57BI6/J wild-type performed during the task (adapted from Papaleo et al., 2008 and 2014; Leggio et al., 2019). 

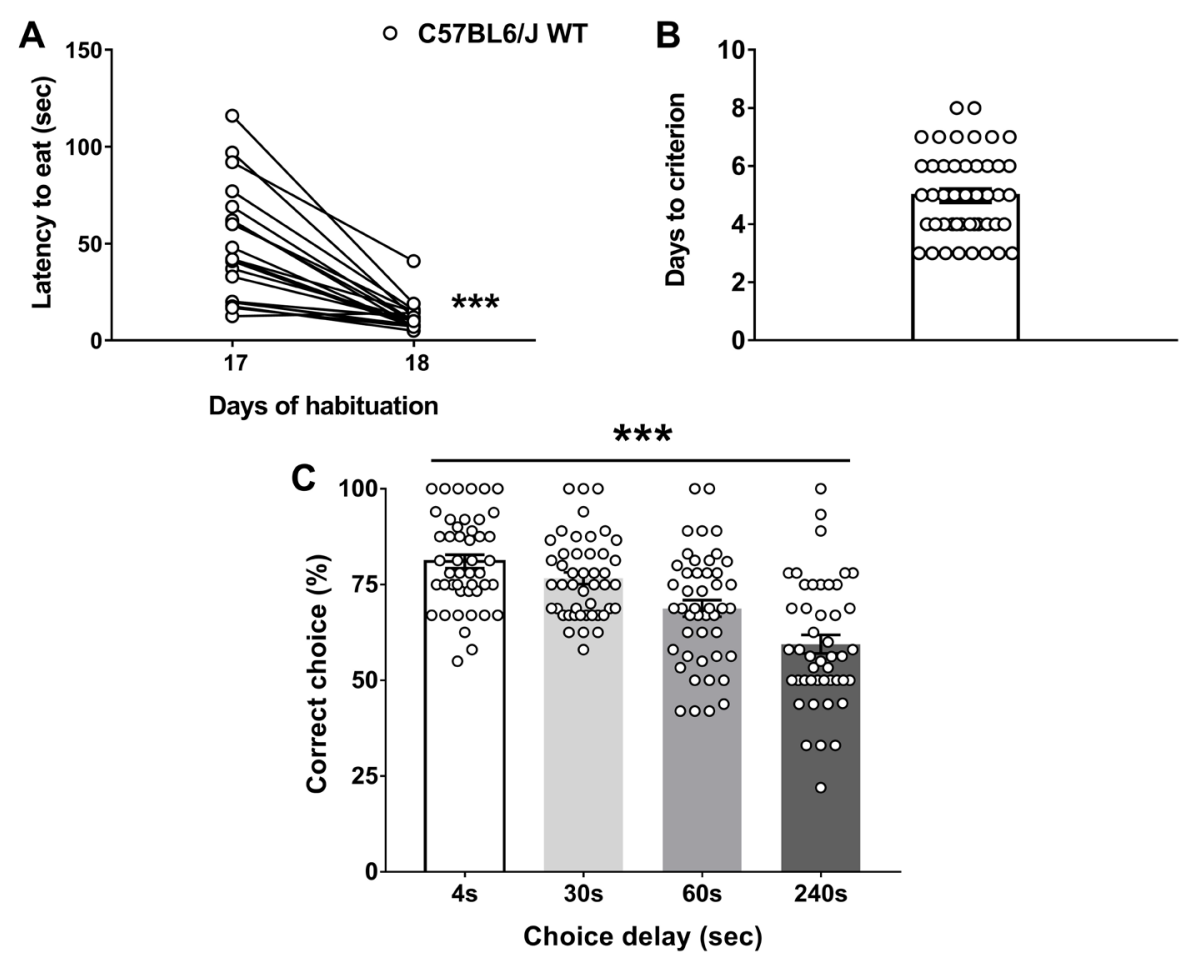

Figure 4. Data distribution of C57BI6/J WT mice $(n=46)$ obtained by clustering different experiments. A. Latency to retrieve the reward (Paired $t$-test; ${ }^{* * *} P<0.001$ ). B. Days needed to reach the criterion. C. Percentage of correct choices with different randomly presented intra-run delays $(4,30,60$, and $240 \mathrm{~s})$ and an inter-trial delay of $20 \mathrm{~s}\left(\right.$ Delay $\mathrm{F}_{(3,180)}=22.49, P<$ 0.0001). The values are the means \pm S.E.M. (adapted from Papaleo et al., 2008 and 2014; Leggio et al., 2019).

\section{Notes}

1. Animals were food restricted throughout the experiment to maintain $90 \%$ of their ad libitum body weight. The maintenance of the $90 \%$ of mice's initial weight is crucial. Mice that do not maintain this body weight condition tend to slow down or do not move into the maze.

2. The ideal number of mice that a researcher should test in this behavioral task is 10-12. Testing more animals in the same experiment could be too challenging because of the long duration of the daily procedure, mostly in the last phase of the test (45-60 min per mouse).

3. During the experiment, researchers should avoid to put the mouse on the grid of the cage. It is less stressful for the mouse picking up it from the home cage and placing it directly into the start position of the maze.

\section{Acknowledgments}

We thank Dr. M. Morini, D. Cantatore, R. Navone, G. Pruzzo, A. Parodi, A. Monteforte and C. Chiabrera for technical support. This work was supported by funding from the Istituto Italiano di 
Please cite this article as: Leggio et. al., (2020). The Discrete Paired-trial Variable-delay T-maze Task to Assess Working Memory in Mice,Bio-protocol 10

Tecnologia, the University of Catania, the Brain and Behavior Research Foundation (2015 NARSAD 23234), and the Compagnia di San Paolo (2015-0321). The methodology described was previously used in Leggio et al. (2019).

\section{Competing interests}

The authors declare that they have no competing interests.

\section{Ethics}

Animal sample size was chosen based on studies using related methods and is similar to what is generally employed in the field. Randomisation was not used to assign animals to experimental groups, and the investigator was blinded to the genotype of animals. All experiments were carried out according to EU Directive 2010/63/EU and the Institutional Animal Care and Use Committees of both Catania University and the Istituto Italiano di Tecnologia (IIT).

\section{References}

1. Aultman, J. M. and Moghaddam, B. (2001). Distinct contributions of glutamate and dopamine receptors to temporal aspects of rodent working memory using a clinically relevant task. Psychopharmacology (Berl) 153(3):353-364.

2. Baddeley, A. (2010). Working memory. Curr Biol 20(4): R136-140.

3. Barch, D. M., Moore, H., Nee, D. E., Manoach, D. S. and Luck, S. J. (2012). CNTRICS imaging biomarkers selection: Working memory. Schizophr Bull 38(1): 43-52.

4. Callicott, J. H., Bertolino, A., Mattay, V. S., Langheim, F. J., Duyn, J., Coppola, R., Goldberg, T. E. and Weinberger, D. R. (2000). Physiological dysfunction of the dorsolateral prefrontal cortex in schizophrenia revisited. Cereb Cortex 10(11): 1078-1092.

5. Castner, S. A., Goldman-Rakic, P. S. and Williams, G. V. (2004). Animal models of working memory: insights for targeting cognitive dysfunction in schizophrenia. Psychopharmacology (Berl) 174(1): 111-125.

6. Dudchenko, P. A. (2004). An overview of the tasks used to test working memory in rodents. Neurosci Biobehav Rev 28(7): 699-709.

7. Dunnett, S. B. (1993). The role and repair of forebrain cholinergic systems in short-term memory. Studies using the delayed matching-to-position task in rats. Adv Neurol 59: 53-65.

8. Floresco, S. B., Seamans, J. K. and Phillips, A. G. (1997). Selective roles for hippocampal, prefrontal cortical, and ventral striatal circuits in radial-arm maze tasks with or without a delay. $J$ Neurosci 17(5): 1880-1890.

9. Forbes, N. F., Carrick, L. A., McIntosh, A. M. and Lawrie, S. M. (2009). Working memory in schizophrenia: a meta-analysis. Psychol Med 39(6): 889-905. 
Please cite this article as: Leggio et. al., (2020). The Discrete Paired-trial Variable-delay T-maze Task to Assess Working Memory in Mice,Bio-protocol 10 (13): e3664. DOI: 10.21769/BioProtoc.3664.

10. Ji, Y., Yang, F., Papaleo, F., Wang, H.X., Gao, W.J., Weinberger, D.R. and Lu, B. (2009). Role of dysbindin in dopamine receptor trafficking and cortical GABA function. Proc Natl Acad Sci U S A 106(46): 19593-19598.

11. Kellendonk, C., Simpson, E. H., Polan, H. J., Malleret, G., Vronskaya, S., Winiger, V., Moore, H. and Kandel, E. R. (2006). Transient and selective overexpression of dopamine D2 receptors in the striatum causes persistent abnormalities in prefrontal cortex functioning. Neuron 49(4): 603-615.

12. Leggio, G. M., Torrisi, S. A., Mastrogiacomo, R., Mauro, D., Chisari, M., Devroye, C., Scheggia, D., Nigro, M., Geraci, F., Pintori, N., Giurdanella, G., Costa, L., Bucolo, C., Ferretti, V., Sortino, M. A., Ciranna, L., De Luca, M. A., Mereu, M., Managò, F., Salomone, S., Drago, F. and Papaleo, F. (2019). The epistatic interaction between the dopamine D3 receptor and dysbindin-1 modulates higher-order cognitive functions in mice and humans. Mol Psychiatry Sep 6.

13. Morris, R.G., Garrud, P., Rawlins, J. N. and O'Keefe, J. (1982). Place navigation impaired in rats with hippocampal lesions. Nature 297(5868): 681-683.

14. Myroshnychenko, M., Seamans, J. K., Phillips, A. G. and Lapish, C. C. (2017). Temporal Dynamics of Hippocampal and Medial Prefrontal Cortex Interactions During the Delay Period of a Working Memory-Guided Foraging Task. Cereb Cortex 27(11): 5331-5342.

15. Papaleo, F., Burdick, M. C., Callicott, J. H. and Weinberger, D. R. (2014). Epistatic interaction between COMT and DTNBP1 modulates prefrontal function in mice and in humans. Mol Psychiatry 19(3):311-316.

16. Papaleo, F., Crawley, J. N., Song, J., Lipska, B. K., Pickel, J., Weinberger, D. R. and Chen, J. (2008). Genetic dissection of the role of catechol-O-methyltransferase in cognition and stress reactivity in mice. $J$ Neurosci 28(35): 8709-8723.

17. Papaleo, F., Yang, F., Garcia, S., Chen, J., Lu, B., Crawley, J. N. and Weinberger, D. R. (2012). Dysbindin-1 modulates prefrontal cortical activity and schizophrenia-like behaviors via dopamine/D2 pathways. Mol Psychiatry 17(1): 85-98.

18. Scheggia, D., Manago, F., Maltese, F., Bruni, S., Nigro, M., Dautan, D., Latuske, P., Contarini, G., Gomez-Gonzalo, M., Requie, L. M., Ferretti, V., Castellani, G., Mauro, D., Bonavia, A., Carmignoto, G., Yizhar, O. and Papaleo, F. (2020). Somatostatin interneurons in the prefrontal cortex control affective state discrimination in mice. Nat Neurosci 23(1): 47-60.

19. Scheggia, D., Mastrogiacomo, R., Mereu, M., Sannino, S., Straub, R. E., Armando, M., Managò, F., Guadagna, S., Piras, F., Zhang, F., Kleinman, J. E., Hyde, T. M., Kaalund, S. S., Pontillo, M., Orso, G., Caltagirone, C., Borrelli, E., De Luca, M. A., Vicari, S., Weinberger, D. R., Spalletta, G. and Papaleo, F. (2018). Variations in Dysbindin-1 are associated with cognitive response to antipsychotic drug treatment. Nat Commun 9(1): 2265.

20. Seamans, J. K., Floresco, S. B. and Phillips, A. G. (1995). Functional differences between the prelimbic and anterior cingulate regions of the rat prefrontal cortex. Behav Neurosci 109(6): 1063-1073. 
Please cite this article as: Leggio et. al., (2020). The Discrete Paired-trial Variable-delay T-maze Task to Assess Working Memory in Mice,Bio-protocol 10 (13): e3664. DOI: 10.21769/BioProtoc.3664.

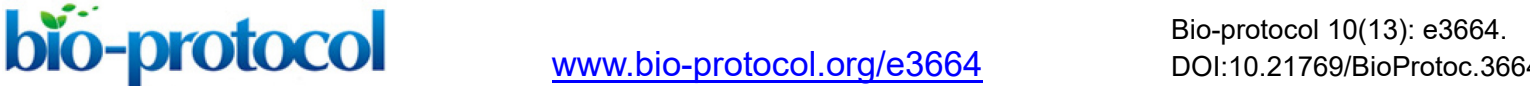

21. Seamans, J. K. and Phillips, A. G. (1994). Selective memory impairments produced by transient lidocaine-induced lesions of the nucleus accumbens in rats. Behav Neurosci 108(3): 456-68.

22. Wager, T. D. and Smith, E. E. (2003). Neuroimaging studies of working memory: a meta-analysis. Cogn Affect Behav Neurosci 3(4): 255-274.

23. Young, J. W., Kerr, L. E., Kelly, J. S., Marston, H. M., Spratt, C., Finlayson, K. and Sharkey, J. (2007). The odour span task: a novel paradigm for assessing working memory in mice. Neuropharmacology 52(2): 634-45. 